\title{
EXISTENCE RESULTS FOR SEMILINEAR PERTURBED FUNCTIONAL DIFFERENTIAL EQUATIONS WITH NONDENSELY DEFINED OPERATORS
}

\author{
M. BELMEKKI, M. BENCHOHRA, AND S. K. NTOUYAS
}

Received 24 January 2006; Revised 9 August 2006; Accepted 5 September 2006

We will establish sufficient conditions for the existence of integral solutions and extremal integral solutions for semilinear functional differential equations with nondensely defined operators in Banach spaces.

Copyright (c) 2006 M. Belmekki et al. This is an open access article distributed under the Creative Commons Attribution License, which permits unrestricted use, distribution, and reproduction in any medium, provided the original work is properly cited.

\section{Introduction}

This paper is concerned with the existence of integral solutions and extremal integral solutions defined on a compact real interval for first-order semilinear differential equations. In Section 3, we consider the following class of semilinear differential equations:

$$
\begin{gathered}
y^{\prime}(t)-A y(t)=f\left(t, y_{t}\right)+g\left(t, y_{t}\right), \quad t \in J:=[0, T], \\
y(t)=\phi(t), \quad t \in[-r, 0],
\end{gathered}
$$

where $f, g: J \times C([-r, 0], E) \rightarrow E$ are given functions, $A: D(A) \subset E \rightarrow E$ is a nondensely defined closed linear operator on $E, \phi:[-r, 0] \rightarrow E$ a given continuous function, and $(E,|\cdot|)$ a real Banach space.

For any function $y$ defined on $[-r, T]$ and any $t \in J$, we denote by $y_{t}$ the element of $C([-r, 0], E)$ defined by

$$
y_{t}(\theta)=y(t+\theta), \quad \theta \in[-r, 0]
$$

Here $y_{t}(\cdot)$ represents the history of the state from time $t-r$, up to the present time $t$.

There has been extensive study of semilinear functional differential equations, where the operator $A$ generates a $C_{0}$ semigroup, or equivalently, when a closed linear operator 


\section{Perturbed functional differential equations}

$A$ satisfies

(i) $\overline{D(A)}=E$,

(ii) the Hille-Yosida condition, that is, there exist $M \geq 0$ and $\tau \in \mathbb{R}$ such that

$$
(\tau, \infty) \subset \rho(A), \quad \sup \left\{(\lambda I-\tau)^{n}\left|(\lambda I-A)^{-n}\right|: \lambda>\tau, n \in \mathbb{N}\right\} \leq M,
$$

where $\rho(A)$ is the resolvent set of $A$ and $I$ is the identity operator. Existence and uniqueness, among other things, are derived. See, for example, the books of Heikkila and Lakshmikantham [9], Kamenskii et al. [10] and the references therein, and the paper by Byszewski and Akca [4].

However, as indicated in [5], we sometimes need to deal with nondensely defined operators. For example, when we look at a one-dimensional heat equation with Dirichlet conditions on $[0,1]$ and consider $A=\partial^{2} / \partial x^{2}$ in $C([0,1], \mathbb{R})$ in order to measure the solutions in the sup-norm, then the domain

$$
D(A)=\left\{\phi \in C^{2}([0,1], \mathbb{R}): \phi(0)=\phi(1)=0\right\}
$$

is not dense in $C([0,1], \mathbb{R})$ with the sup-norm. See [5] for more examples and remarks concerning nondensely defined operators. Recently, evolution functional differential equations with nondensely defined linear operators have received much attention (see, e.g., the papers by Adimy and Ezzinbi [1], Ezzinbi and Liu [7]). Our main results extend similar problems considered in the above-listed papers to nondensely defined operators and where a perturbation term $g$ is considered. Our approach is based on a new fixed point theorem of Burton and Kirk [3]. In Section 4, we will prove the existence of extremal integral solutions of the problem (1.1)-(1.2), and our approach here is based on the concept of upper and lower solutions combined with a fixed point theorem on ordered Banach spaces established recently by Dhage and Henderson [6]. Finally, Section 5 is devoted to an example illustrating the abstract theory considered in the previous sections.

\section{Preliminaries}

In this section, we introduce notations, definitions, and preliminary facts which are used throughout this paper. $C(J, E)$ is the Banach space of all continuous functions from $J$ into $E$ with the norm

$$
\|y\|_{\infty}=\sup \{|y(t)|: t \in J\}
$$

and $C([-r, 0], E)$ is the Banach space of all continuous functions from $[-r, 0]$ into $E$ endowed with the norm $\|\cdot\|$ defined by

$$
\|\phi\|=\sup \{|\phi(\theta)|:-r \leq \theta \leq 0\} .
$$

Also $B(E)$ denotes the Banach space of bounded linear operators from $E$ into $E$ with the norm

$$
\|N\|_{B(E)}=\sup \{|N(y)|:|y|=1\} .
$$


$L^{1}(J, E)$ denotes the Banach space of measurable functions $y: J \rightarrow E$ which are Bochner integrable normed by

$$
\|y\|_{L^{1}}=\int_{0}^{T}|y(t)| d t
$$

Definition 2.1 [2]. Let $E$ be a Banach space. An integrated semigroup is a family of operators $(S(t))_{t \geq 0}$ of bounded linear operators $S(t)$ on $E$ with the following properties:

(i) $S(0)=0$;

(ii) $t \rightarrow S(t)$ is strongly continuous;

(iii) $S(s) S(t)=\int_{0}^{s}(S(t+r)-S(r)) d r$ for all $t, s \geq 0$.

Definition 2.2. An integrated semigroup $(S(t))_{t \geq 0}$ is called exponential bounded, if there exist constants $M \geq 0$ and $\omega \in \mathbb{R}$ such that

$$
|S(t)| \leq M e^{\omega t}, \quad \text { for } t \geq 0 \text {. }
$$

Moreover, $(S(t))_{t \geq 0}$ is called nondegenerate if $S(t) x=0$, for all $t \geq 0$, implies $x=0$.

Definition 2.3. An operator $A$ is called a generator of an integrated semigroup, if there exists $\omega \in \mathbb{R}$ such that $(\omega,+\infty) \subset \rho(A)$, and there exists a strongly continuous exponentially bounded family $(S(t))_{t \geq 0}$ of linear bounded operators such that $S(0)=0$ and $(\lambda I-A)^{-1}=\lambda \int_{0}^{\infty} e^{-\lambda t} S(t) d t$ for all $\lambda>\omega$.

If $A$ is the generator of an integrated semigroup $(S(t))_{t \geq 0}$ which is locally Lipschitz, then from [2], $S(\cdot) x$ is continuously differentiable if and only if $x \in \overline{D(A)}$. In particular, $S^{\prime}(t) x:=(d / d t) S(t) x$ defines a bounded operator on the set $E_{1}:=\{x \in E: t \rightarrow S(t) x$ is continously differentiable on $[0, \infty)\}$ and $\left(S^{\prime}(t)\right)_{t \geq 0}$ is a $C_{0}$ semigroup on $\overline{D(A)}$. Here and hereafter, we assume that $A$ satisfies the Hille-Yosida condition, that is, there exist $M \geq 0$ and $\omega \in \mathbb{R}$ such that $(\omega, \infty) \subset \rho(A)$, sup $\left\{(\lambda I-\omega)^{n}\left|(\lambda I-A)^{-n}\right|: \lambda>\omega, n \in \mathbb{N}\right\} \leq M$.

Let $(S(t))_{t \geq 0}$ be the integrated semigroup generated by $A$. We note that, since $A$ satisfies the Hille-Yosida condition, $\left\|S^{\prime}(t)\right\|_{B(E)} \leq M e^{\omega t}, t \geq 0$, where $M$ and $\omega$ are the constants considered in the Hille-Yosida condition (see [11]). lem:

In the sequel, we give some results for the existence of solutions of the following prob-

$$
\begin{gathered}
y^{\prime}(t)=A y(t)+g(t), \quad t \geq 0, \\
y(0)=a \in E,
\end{gathered}
$$

where $A$ satisfies the Hille-Yosida condition, without being densely defined.

Theorem $2.4[11]$. Let $g:[0, b] \rightarrow$ E be a continuous function. Then for $a \in \overline{D(A)}$, there exists a unique continuous function $y:[0, b] \rightarrow$ E such that

(i) $\int_{0}^{t} y(s) d s \in D(A)$ for $t \in[0, b]$,

(ii) $y(t)=a+A \int_{0}^{t} y(s) d s+\int_{0}^{t} g(s) d s, t \in[0, b]$,

(iii) $|y(t)| \leq M e^{\omega t}\left(|a|+\int_{0}^{t} e^{-\omega s}|g(s)| d s\right), t \in[0, b]$. 
4 Perturbed functional differential equations

Moreover, $y$ is given by the following variation of constants formula:

$$
y(t)=S^{\prime}(t) a+\frac{d}{d t} \int_{0}^{t} S(t-s) g(s) d s, \quad t \geq 0
$$

Let $B_{\lambda}=\lambda R(\lambda, A):=\lambda(\lambda I-A)^{-1}$. Then (see [11]), for all $x \in \overline{D(A)}, B_{\lambda} x \rightarrow x$ as $\lambda \rightarrow \infty$. Also from the Hille-Yosida condition (with $n=1$ ), it is easy to see that $\lim _{\lambda \rightarrow \infty}\left|B_{\lambda} x\right| \leq$ $M|x|$, since

$$
\left|B_{\lambda}\right|=\left|\lambda(\lambda I-A)^{-1}\right| \leq \frac{M \lambda}{\lambda-\omega} .
$$

Thus $\lim _{\lambda \rightarrow \infty}\left|B_{\lambda}\right| \leq M$. Also if $y$ is given by (2.8), then

$$
y(t)=S^{\prime}(t) a+\lim _{\lambda \rightarrow \infty} \int_{0}^{t} S^{\prime}(t-s) B_{\lambda} g(s) d s, \quad t \geq 0 .
$$

Definition 2.5. The map $f: J \times C([-r, 0], E) \rightarrow E$ is said to be $L^{1}$-Carathéodory if

(i) $t \mapsto f(t, u)$ is measurable for each $u \in C([-r, 0], E)$;

(ii) $u \mapsto f(t, u)$ is continuous for almost all $t \in J$;

(iii) for each $q>0$, there exists $\varphi_{q} \in L^{1}\left(J, \mathbb{R}_{+}\right)$such that

$$
|f(t, u)| \leq \varphi_{q}(t) \quad \forall\|u\| \leq q \text { and for a.e. } t \in J .
$$

\section{Existence of integral solutions}

Now, we are able to state and prove our main theorem for the initial value problem (1.1)(1.2). Before starting and proving this one, we give the definition of its integral solution.

Definition 3.1. Say that $y:[-r, T] \rightarrow E$ is an integral solution of (1.1)-(1.2) if

(i) $y(t)=\phi(0)+A \int_{0}^{t} y(s) d s+\int_{0}^{t} f\left(s, y_{s}\right) d s+\int_{0}^{t} g\left(s, y_{s}\right) d s, t \in J$;

(ii) $\int_{0}^{t} y(s) d s \in D(A)$ for $t \in J$, and $y(t)=\phi(t), t \in[-r, 0]$.

From the definition, it follows that $y(t) \in \overline{D(A)}$, for all $t \geq 0$, in particular $\phi(0) \in$ $\overline{D(A)}$. Moreover, $y$ satisfies the following variation of constants formula:

$$
y(t)=S^{\prime}(t) \phi(0)+\frac{d}{d t} \int_{0}^{t} S(t-s) f\left(s, y_{s}\right) d s+\frac{d}{d t} \int_{0}^{t} S(t-s) g\left(s, y_{s}\right) d s, \quad t \geq 0
$$

We notice also that, if $y$ satisfies (3.1), then

$$
y(t)=S^{\prime}(t) \phi(0)+\lim _{\lambda \rightarrow \infty} \int_{0}^{t} S^{\prime}(t-s) B_{\lambda}\left[f\left(s, y_{s}\right)+g\left(s, y_{s}\right)\right] d s, \quad t \geq 0 .
$$

Our main result in this section is based upon the following fixed point theorem due to Burton and Kirk [3]. 
Theorem 3.2. Let $X$ be a Banach space, and $A, B$ two operators satisfying

(i) $A$ is a contraction, and

(ii) $B$ is completely continuous.

Then either

(a) the operator equation $y=A(y)+B(y)$ has a solution, or

(b) the set $\mathscr{E}=\{u \in X: \lambda A(u / \lambda)+\lambda B(u)=u\}$ is unbounded for $\lambda \in(0,1)$.

Our main result reads.

Theorem 3.3. Assume that

(H1) A satisfies Hille-Yosida condition;

(H2) the function $f: J \times C([-r, 0], E) \rightarrow E$ is $L^{1}$-Carathéodory;

(H3) the operator $S^{\prime}(t)$ is compact in $\overline{D(A)}$ whenever $t>0$;

(H4) there exists a function $k(t) \in L^{1}\left(J, \mathbb{R}_{+}\right)$such that

$$
|g(t, u)-g(t, \bar{u})| \leq k(t)\|u-\bar{u}\|, \quad \text { for a.e. } t \in J, u, \bar{u} \in C([-r, 0], E),
$$

with

$$
M e^{\omega T} \int_{0}^{T} e^{-\omega s} k(s) d s<1
$$

(H5) there exists a function $p \in L^{1}\left(J, \mathbb{R}_{+}\right)$and a continuous nondecreasing function $\psi$ : $[0, \infty) \rightarrow[0, \infty)$ such that

$$
|f(t, u)| \leq p(t) \psi(\|u\|), \quad \text { for a.e. } t \in J, \quad \text { and each } u \in C([-r, 0], E)
$$

with

$$
\int_{c}^{\infty} \frac{d s}{s+\psi(s)}>\|m\|_{L^{1}}
$$

where

$$
\begin{aligned}
& c=M\|\phi\|+M \int_{0}^{T} e^{-\omega s}|g(s, 0)| d s, \\
& m(t)=\max \left\{\omega^{*}+M k(t), M p(t)\right\},
\end{aligned}
$$

and $\omega^{*}=\omega$ if $\omega>0$ and $\omega^{*}=0$ if $\omega<0$.

Then, if $\phi(0) \in \overline{D(A)}$, the initial value problem (IVP for short) (1.1)-(1.2) has at least one integral solution on $[-r, T]$.

Proof. Transform the IVP (1.1)-(1.2) into a fixed point problem. Consider the two operators

$$
F, G: C([-r, T], E) \longrightarrow C([-r, T], E)
$$


6 Perturbed functional differential equations

defined by

$$
\begin{aligned}
& F(y)(t)= \begin{cases}\phi(t), & t \in[-r, 0], \\
S^{\prime}(t) \phi(0)+\frac{d}{d t} \int_{0}^{t} S(t-s) f\left(s, y_{s}\right) d s, & t \in J,\end{cases} \\
& G(y)(t)= \begin{cases}0, & t \in[-r, 0], \\
\frac{d}{d t} \int_{0}^{t} S(t-s) g\left(s, y_{s}\right) d s, & t \in J .\end{cases}
\end{aligned}
$$

Then the problem of finding the solution of IVP (1.1)-(1.2) is reduced to finding the solution of the operator equation $F(y)(t)+G(y)(t)=y(t), t \in[-r, T]$. We will show that the operators $F$ and $G$ satisfy all conditions of Theorem 3.2. The proof will be given in several steps.

Step 1. F is continuous.

Let $\left\{y_{n}\right\}$ be a sequence such that $y_{n} \rightarrow y$ in $C([-r, T], E)$. Then for $\omega>0$ (if $\omega<0$ it is $\left.e^{\omega t}<1\right)$,

$$
\begin{aligned}
\left|F\left(y_{n}\right)(t)-F(y)(t)\right| & =\left|\frac{d}{d t} \int_{0}^{t} S(t-s)\left[f\left(s, y_{n_{s}}\right)-f\left(s, y_{s}\right)\right] d s\right| \\
& \leq M e^{\omega T} \int_{0}^{T} e^{-\omega s}\left|f\left(s, y_{n_{s}}\right)-f\left(s, y_{s}\right)\right| d s .
\end{aligned}
$$

Since $f(s, \cdot)$ is continuous, we have by the Lebesgue dominated convergence theorem

$$
\left\|F\left(y_{n}\right)(t)-F(y)(t)\right\|_{\infty} \leq M e^{\omega T}\left\|f\left(\cdot, y_{n .}\right)-f(\cdot, y .)\right\|_{L^{1}} \longrightarrow 0, \quad \text { as } n \longrightarrow \infty .
$$

Thus $F$ is continuous.

Step 2. F maps bounded sets into bounded sets in $C([-r, T], E)$.

It is enough to show that for any $q>0$ there exists a positive constant $l$ such that for each $y \in B_{q}=\left\{y \in C([-r, T], E):\|y\|_{\infty} \leq q\right\}$ we have $F(y) \in B_{l}$.

Then we have for each $t \in J$,

$$
\begin{aligned}
|F(y)(t)| & =\left|S^{\prime}(t) \phi(0)+\frac{d}{d t} \int_{0}^{t} S(t-s) f\left(s, y_{s}\right) d s\right| \\
& \leq M e^{\omega T}|\phi(0)|+M e^{\omega T} \int_{0}^{T} e^{-\omega s} \varphi_{q}(s) d s
\end{aligned}
$$

here $\varphi_{q}$ is chosen as in Definition 2.5. Then we have

$$
\|F(y)(t)\|_{\infty} \leq M e^{\omega T}\|\phi\|+M e^{\omega T} \int_{0}^{T} e^{-\omega s} \varphi_{q}(s) d s:=l .
$$

Step 3. F maps bounded sets into equicontinuous sets of $C([-r, T], E)$.

We consider $B_{q}$ as in Step 2 and let $\epsilon>0$ be given. Now let $\tau_{1}, \tau_{2} \in[-r, T]$ with $\tau_{2}>\tau_{1}$. We consider two cases: $\tau_{1}>\epsilon$ and $\tau_{1} \leq \epsilon$. 
Case 1. If $\tau_{1}>\epsilon$, then

$$
\begin{aligned}
\left|F(y)\left(\tau_{2}\right)-F(y)\left(\tau_{1}\right)\right| \leq & \left|S^{\prime}\left(\tau_{2}\right) \phi(0)-S^{\prime}\left(\tau_{1}\right) \phi(0)\right| \\
& +\left|\lim _{\lambda \rightarrow \infty} \int_{0}^{\tau_{1}-\epsilon}\left[S^{\prime}\left(\tau_{2}-s\right)-S^{\prime}\left(\tau_{1}-s\right)\right] B_{\lambda} f\left(s, y_{s}\right) d s\right| \\
& +\left|\lim _{\lambda \rightarrow \infty} \int_{\tau_{1}-\epsilon}^{\tau_{1}}\left[S^{\prime}\left(\tau_{2}-s\right)-S^{\prime}\left(\tau_{1}-s\right)\right] B_{\lambda} f\left(s, y_{s}\right) d s\right| \\
& +\left|\lim _{\lambda \rightarrow \infty} \int_{\tau_{1}}^{\tau_{2}} S^{\prime}\left(\tau_{2}-s\right) B_{\lambda} f\left(s, y_{s}\right) d s\right| \\
\leq & \left|S^{\prime}\left(\tau_{2}\right) \phi(0)-S^{\prime}\left(\tau_{1}\right) \phi(0)\right| \\
& +M^{*}|| S^{\prime}\left(\tau_{2}-\tau_{1}+\epsilon\right)-S^{\prime}(\epsilon) \|_{B(E)} \int_{0}^{\tau_{1}-\epsilon} e^{-\omega s} \varphi_{q}(s) d s \\
& +2 M^{*} \int_{\tau_{1}-\epsilon}^{\tau_{1}} e^{-\omega s} \varphi_{q}(s) d s+M^{*} \int_{\tau_{1}}^{\tau_{2}} e^{-\omega s} \varphi_{q}(s) d s ;
\end{aligned}
$$

here $M^{*}=M \max \left\{e^{\omega T}, 1\right\}$.

Case 2. Let $\tau_{1} \leq \epsilon$. For $\tau_{2}-\tau_{1}<\epsilon$, we get

$$
\begin{aligned}
& \left|F(y)\left(\tau_{2}\right)-F(y)\left(\tau_{1}\right)\right| \\
& \quad \leq\left|S^{\prime}\left(\tau_{2}\right) \phi(0)-S^{\prime}\left(\tau_{1}\right) \phi(0)\right|+M^{*} \int_{0}^{2 \epsilon} e^{-\omega s} \varphi_{q}(s) d s+M^{*} \int_{0}^{\epsilon} e^{-\omega s} \varphi_{q}(s) d s .
\end{aligned}
$$

Note that equicontinuity follows since (i) $S^{\prime}(t), t \geq 0$, is a strongly continuous semigroup and (ii) $S^{\prime}(t)$ is compact for $t>0$ ( $s o S^{\prime}(t)$ is continuous in the uniform operator topology for $t>0)$.

Let $0<t \leq T$ be fixed and let $\epsilon$ be a real number satisfying $0<\epsilon<t$. For $y \in B_{q}$, we define

$$
\begin{aligned}
F_{\epsilon}(y)(t) & =S^{\prime}(t) \phi(0)+\lim _{\lambda \rightarrow \infty} \int_{0}^{t-\epsilon} S^{\prime}(t-s) B_{\lambda} f\left(s, y_{s}\right) d s \\
& =S^{\prime}(t) \phi(0)+S^{\prime}(\epsilon) \lim _{\lambda \rightarrow \infty} \int_{0}^{t-\epsilon} S^{\prime}(t-s-\epsilon) B_{\lambda} f\left(s, y_{s}\right) d s .
\end{aligned}
$$

Note that

$$
\left\{\lim _{\lambda \rightarrow \infty} \int_{0}^{t-\epsilon} S^{\prime}(t-s-\epsilon) B_{\lambda} f\left(s, y_{s}\right) d s: y \in B_{q}\right\}
$$

is a bounded set since

$$
\left|\lim _{\lambda \rightarrow \infty} \int_{0}^{t-\epsilon} S^{\prime}(t-s-\epsilon) B_{\lambda} f\left(s, y_{s}\right) d s\right| \leq M^{*} \int_{0}^{t-\epsilon} e^{-\omega s} \varphi_{q}(s) d s
$$


and now since $S^{\prime}(t)$ is a compact operator for $t>0$, the set $Y_{\varepsilon}(t)=\left\{F_{\varepsilon}(y)(t): y \in B_{q}\right\}$ is relatively compact in $E$ for every $\varepsilon, 0<\varepsilon<t$. Moreover,

$$
\left|F(y)(t)-F_{\varepsilon}(y)(t)\right| \leq M^{*} \int_{t-\varepsilon}^{t} e^{-\omega s} \varphi_{q}(s) d s .
$$

Therefore, the set $Y(t)=\left\{F(y)(t): y \in B_{q}\right\}$ is totally bounded. Hence $Y(t)$ is relatively compact in $E$.

As a consequence of Steps 2 and 3 and the Arzelá-Ascoli theorem, we can conclude that $F: C([-r, T], E) \rightarrow C([-r, T], E)$ is a completely continuous operator.

Step 4. $G$ is a contraction.

Let $x, y \in C([-r, T], E)$. Then

$$
\begin{aligned}
|G(x)(t)-G(y)(t)| & =\left|\frac{d}{d t} \int_{0}^{t} S(t-s)\left[g\left(s, x_{s}\right)-g\left(s, y_{s}\right)\right] d s\right| \\
& \leq M e^{\omega T} \int_{0}^{T} e^{-\omega s}\left|g\left(s, x_{s}\right)-g\left(s, y_{s}\right)\right| d s \\
& \leq M e^{\omega T} \int_{0}^{T} e^{-\omega s} k(s)|| x_{s}-y_{s}|| d s .
\end{aligned}
$$

Then

$$
\|G(x)-G(y)\|_{\infty} \leq\left(M e^{\omega T} \int_{0}^{T} e^{-\omega s} k(s) d s\right)\|x-y\|_{\infty},
$$

which is a contraction, since $M e^{\omega T} \int_{0}^{T} e^{-\omega s} k(s) d s<1$, by condition (3.4).

Step 5. A priori bounds.

Now it remains to show that the set

$$
\mathscr{E}=\left\{y \in C([-r, T], E): y=\lambda F(y)+\lambda G\left(\frac{y}{\lambda}\right) \text { for some } 0<\lambda<1\right\}
$$

is bounded.

Let $y \in \mathscr{E}$. Then $y=\lambda F(y)+\lambda G(y / \lambda)$ for some $0<\lambda<1$. Thus, for each $t \in J$,

$$
y(t)=\lambda S^{\prime}(t) \phi(0)+\lambda \frac{d}{d t} \int_{0}^{t} S(t-s) f\left(s, y_{s}\right) d s+\lambda \frac{d}{d t} \int_{0}^{t} S(t-s) g\left(s, \frac{y_{s}}{\lambda}\right) d s .
$$

This implies by (H5) that, for each $t \in J$, we have

$$
\begin{aligned}
|y(t)| \leq & \lambda M e^{\omega t}|\phi(0)|+\lambda M e^{\omega t} \int_{0}^{t} e^{-\omega s} p(s) \psi\left(\left\|y_{s}\right\|\right) d s \\
& +\lambda M e^{\omega t} \int_{0}^{t} e^{-\omega s}\left|g\left(s, \frac{y_{s}}{\lambda}\right)-g(s, 0)\right| d s+\lambda M e^{\omega t} \int_{0}^{t} e^{-\omega s}|g(s, 0)| d s \\
\leq & M e^{\omega t}\|\phi\|+M e^{\omega t} \int_{0}^{t} e^{-\omega s} p(s) \psi\left(\left\|y_{s}\right\|\right) d s \\
& +M e^{\omega t} \int_{0}^{t} e^{-\omega s} k(s)\left\|y_{s}\right\| d s+M e^{\omega t} \int_{0}^{t} e^{-\omega s}|g(s, 0)| d s .
\end{aligned}
$$


We consider the function $\mu$ defined by

$$
\mu(t)=\sup \{|y(s)|:-r \leq s \leq t\}, \quad 0 \leq t \leq T .
$$

Consider the case $\omega>0$; the case $\omega<0$ is more easy, since $e^{\omega t}<1$. Let $t^{*} \in[-r, t]$ be such that $\mu(t)=\left|y\left(t^{*}\right)\right|$. If $t^{*} \in[0, T]$, by the previous inequality, we have for $t \in[0, T]$ (note that $\left.t^{*} \leq t\right)$

$e^{-\omega t} \mu(t) \leq M\|\phi\|+M \int_{0}^{t} e^{-\omega s} p(s) \psi(\mu(s)) d s+M \int_{0}^{t} e^{-\omega s} k(s) \mu(s) d s+M \int_{0}^{T} e^{-\omega s}|g(s, 0)| d s$.

If $t^{*} \in[-r, 0]$, then $\mu(t) \leq\|\phi\|$ and the previous inequality holds.

Let us take the right-hand side of (3.27) as $v(t)$. Then we have

$$
\begin{gathered}
\mu(t) \leq e^{\omega t} v(t) \quad \forall t \in J, \\
v(0)=M\|\phi\|+M \int_{0}^{T} e^{-\omega s}|g(s, 0)| d s, \\
v^{\prime}(t)=M e^{-\omega t} p(t) \psi(\mu(t))+M k(t) e^{-\omega t} \mu(t), \quad \text { a.e. } t \in J .
\end{gathered}
$$

Using the nondecreasing character of $\psi$, we get

$$
v^{\prime}(t) \leq M e^{-\omega t} p(t) \psi\left(e^{\omega t} v(t)\right)+M k(t) v(t), \quad \text { a.e. } t \in J .
$$

Then for a.e. $t \in J$, we have

$$
\begin{aligned}
\left(e^{\omega t} v(t)\right)^{\prime} & =\omega e^{\omega t} v(t)+v^{\prime}(t) e^{\omega t} \\
& \leq \omega e^{\omega t} v(t)+M p(t) \psi\left(e^{\omega t} v(t)\right)+M k(t) e^{\omega t} v(t) \\
& \leq m(t)\left[e^{\omega t} v(t)+\psi\left(e^{\omega t} v(t)\right)\right] .
\end{aligned}
$$

Thus

$$
\int_{v(0)}^{e^{\omega t} v(t)} \frac{d u}{u+\psi(u)} \leq \int_{0}^{T} m(s) d s=\|m\|_{L^{1}}<\int_{c}^{\infty} \frac{d u}{u+\psi(u)} .
$$

Consequently, by condition (3.6), there exists a constant $d$ such that $e^{\omega t} v(t) \leq d, t \in J$, and hence $\|y\|_{\infty} \leq d$ where $d$ depends only on the constants $M, \omega$ and the functions $p, k$, and $\psi$. This shows that the set $\mathscr{E}$ is bounded. As a consequence of Theorem 3.2, we deduce that $F(y)+G(y)$ has a fixed point which is an integral solution of problem (1.1)-(1.2).

\section{Existence of extremal integral solutions}

In this section, we will prove the existence of maximal and minimal integral solutions of IVP (1.1)-(1.2) under suitable monotonicity conditions on the functions involved in it. 
Definition 4.1. A nonempty closed subset $C$ of a Banach space $X$ is said to be a cone if

(i) $C+C \subset C$,

(ii) $\lambda C \subset C$ for $\lambda>0$, and

(iii) $\{-C\} \cap\{C\}=\{0\}$.

A cone $C$ is called normal if the norm $\|\cdot\|$ is semimonotone on $C$, that is, there exists a constant $N>0$ such that $\|x\| \leq N\|y\|$, whenever $x \leq y$. We equip the space $X=C(J, E)$ with the order relation $\leq$ induced by a cone $C$ in $E$, that is, for all $y, \bar{y} \in X: y \leq \bar{y}$ if and only if $\bar{y}(t)-y(t) \in C$, for all $t \in J$. In what follows, will assume that the cone $C$ is normal. Cones and their properties are detailed in $[8,9]$. Let $a, b \in X$ be such that $a \leq b$. Then, by an order interval $[a, b]$, we mean a set of points in $X$ given by

$$
[a, b]=\{x \in X \mid a \leq x \leq b\}
$$

Definition 4.2. Let $X$ be an ordered Banach space. A mapping $T: X \rightarrow X$ is called isotone increasing if $T(x) \leq T(y)$ for any $x, y \in X$ with $x<y$. Similarly, $T$ is called isotone decreasing if $T(x) \geq T(y)$, whenever $x<y$.

Definition 4.3 [9]. Say that $x \in X$ is the least fixed point of $G$ in $X$ if $x=G x$ and $x \leq y$, whenever $y \in X$ and $y=G y$. The greatest fixed point of $G$ in $X$ is defined similarly by reversing the inequality. If both least and greatest fixed points of $G$ in $X$ exist, call them extremal fixed points of $G$ in $X$.

The following fixed point theorem is due to Heikkila and Lakshmikantham.

Theorem 4.4 [9]. Let $[a, b]$ be an order interval in an order Banach space $X$ and let $Q:[a, b] \rightarrow[a, b]$ be a nondecreasing mapping. If each sequence $\left(Q x_{n}\right) \subset Q([a, b])$ converges, whenever $\left(x_{n}\right)$ is a monotone sequence in $[a, b]$, then the sequence of $Q$-iteration of a converges to the least fixed point $x_{*}$ of $Q$ and the sequence of $Q$-iteration of $b$ converges to the greatest fixed point $x^{*}$ of Q. Moreover,

$$
x_{*}=\min \{y \in[a, b], y \geq Q y\}, \quad x^{*}=\max \{y \in[a, b], y \leq Q y\} .
$$

As a consequence, Dhage, Henderson have proved the following.

Theorem 4.5 [6]. Let $K$ be a cone in a Banach space $X$, let $[a, b]$ be an order interval in $a$ Banach space, and let $B_{1}, B_{2}:[a, b] \rightarrow X$ be two functions satisfying

(a) $B_{1}$ is a contraction,

(b) $B_{2}$ is completely continuous,

(c) $B_{1}$ and $B_{2}$ are strictly monotone increasing, and

(d) $B_{1}(x)+B_{2}(x) \in[a, b]$, for all $x \in[a, b]$.

Further, if the cone $K$ in $X$ is normal, then the equation $x=B_{1}(x)+B_{2}(x)$ has a least fixed point $x_{*}$ and a greatest fixed point $x^{*} \in[a, b]$. Moreover, $x_{*}=\lim _{n \rightarrow \infty} x_{n}$ and $x^{*}=$ $\lim _{n \rightarrow \infty} y_{n}$, where $\left\{x_{n}\right\}$ and $\left\{y_{n}\right\}$ are the sequences in $[a, b]$ defined by

$$
x_{n+1}=B_{1}\left(x_{n}\right)+B_{2}\left(x_{n}\right), \quad x_{0}=a, \quad y_{n+1}=B_{1}\left(y_{n}\right)+B_{2}\left(y_{n}\right), \quad y_{0}=b .
$$


We need the following definitions in the sequel.

Definition 4.6. A function $v \in C([-r, T], E)$ is called a lower integral solution of IVP (1.1)-(1.2) if $v(t) \leq \phi(t), t \in[-r, 0]$ and $v(t) \leq \phi(0)+A \int_{0}^{t} v(s) d s+\int_{0}^{t} f\left(s, v_{s}\right) d s+$ $\int_{0}^{t} g\left(s, v_{s}\right)$, a.e. $t \in J$. Similarly, an upper integral solution $w$ of IVP (1.1)-(1.2) is defined by reversing the order of the above inequalities.

Definition 4.7. An integral solution $x_{M}$ of IVP (1.1)-(1.2) is said to be maximal if for any other integral solution $x$ of IVP (1.1)-(1.2) on $J$, one has that $x(t) \leq x_{M}(t)$ for each $t \in J$. Similarly, a minimal integral solution of IVP (1.1)-(1.2) is defined by reversing the order of the inequalities.

Definition 4.8. A function $f(t, x)$ is called strictly monotone increasing in $x$ a.e. for $t \in J$, if $f(t, x) \leq f(t, y)$ a.e. $t \in J$ for all $x, y \in X$ with $x<y$. Similarly, $f(t, x)$ is called strictly monotone decreasing in $x$ a.e. for $t \in J$, if $f(t, x) \geq f(t, y)$ a.e. $t \in J$ for all $x, y \in X$ with $x<y$.

We consider the following assumptions in the sequel.

(H6) The functions $f(t, y)$ and $g(t, y)$ are strictly monotone increasing in $y$ for almost each $t \in J$.

(H7) $S^{\prime}(t)$ is order-preserving, that is, $S^{\prime}(t)(v) \geq 0$, whenever $v \geq 0$.

(H8) The IVP (1.1)-(1.2) has a lower integral solution $v$ and an upper integral solution $w$ with $v \leq w$.

Theorem 4.9. Assume that assumptions (H1)-(H8) hold. Then IVP (1.1)-(1.2) has a minimal and a maximal integral solutions on $[-r, T]$.

Proof. It can be shown, as in the proof of Theorem 3.3, that $F$ is completely continuous and $G$ is a contraction on $[v, w]$. We will show that $F$ and $G$ are isotone increasing on $[v, w]$. Let $y, \bar{y} \in[a, b]$ be such that $y \leq \bar{y}, y \neq \bar{y}$. Then by (H6) and (H7), we have for each $t \in J$,

$$
\begin{aligned}
F(y)(t) & =S^{\prime}(t) \phi(0)+\frac{d}{d t} \int_{0}^{t} S(t-s) f\left(s, y_{s}\right) d s \\
& \leq S^{\prime}(t) \phi(0)+\frac{d}{d t} \int_{0}^{t} S(t-s) f\left(s, \bar{y}_{s}\right) d s \\
& =F(\bar{y})(t) .
\end{aligned}
$$

Similarly, $G(y) \leq G(\bar{y})$. Therefore, $F$ and $G$ are isotone increasing on $[v, w]$. Finally, let $x \in[v, w]$ be any element. By $(\mathrm{H} 8)$, we deduce that

$$
v \leq F(v)+G(v) \leq F(x)+G(x) \leq F(w)+G(w) \leq w
$$

which shows that $F(x)+G(x) \in[v, w]$ for all $x \in[v, w]$. Thus, the functions $F$ and $G$ satisfy all conditions of Theorem 4.5, and hence IVP (1.1)-(1.2) has a maximal and minimal integral solutions on $[-r, T]$. This completes the proof. 


\section{An example}

Consider the system

$$
\begin{gathered}
\frac{\partial}{\partial t} u(t, x)=-\frac{\partial}{\partial x} u(t, x)+F\left(u_{t}(\cdot, x)\right)+G\left(u_{t}(\cdot, x)\right), \quad t \in[0, T] \\
u(t, 0)=0, \quad t \in[0, T], \\
u(\theta, x)=\phi(\theta, x), \quad \theta \in[-r, 0], x \in[0, l]
\end{gathered}
$$

where $l>0, r>0, \phi \in C([-r, 0], X)$, and $F$ and $G$ are continuous functions from $C([-r, 0], X)$ into $\mathbb{R}$.

To write (5.1)-(5.3) in the form (1.1)-(1.2), we choose

$X=C([0, l], \mathbb{R}), \quad y(t)=u(t, \cdot), \quad f\left(t, y_{t}\right)=F\left(y_{t}(\cdot, x)\right), \quad g\left(t, y_{t}\right)=G\left(y_{t}(\cdot, x)\right)$,

and denote by $A y:=-y^{\prime}$ with domain

$$
D(A)=\left\{u \in C^{1}([0, l], \mathbb{R}): u(0)=0\right\} .
$$

We have

$$
\overline{D(A)}=\{u \in C([0, l], \mathbb{R}): u(0)=0\} \neq X
$$

It is well known (see [5]) that $A$ satisfies the following properties:

(i) $(0, \infty) \subset \rho(A)$;

(ii) $\left\|(\lambda I-A)^{-1}\right\| \leq 1 / \lambda, \lambda>0$.

This implies that the operator $A$ satisfies the Hille-Yosida condition (with $M=1$ and $\omega=0)$. Then problem (5.1)-(5.3) can be written as

$$
\begin{gathered}
u^{\prime}(t)=A u(t)+f\left(t, u_{t}\right)+g\left(t, u_{t}\right), \quad t \in[0, T] \\
u(t)=\phi(t), \quad t \in[-r, 0]
\end{gathered}
$$

Thus, under appropriate conditions on the functions $F$ and $G$ as those in (H1)-(H8), the problem (5.1)-(5.3) has an integral solution as well as extremal integral solutions.

\section{Acknowledgment}

The authors are thankful to the referee for his/her comments and remarks.

\section{References}

[1] M. Adimy and K. Ezzinbi, A class of linear partial neutral functional-differential equations with nondense domain, Journal of Differential Equations 147 (1998), no. 2, 285-332.

[2] W. Arendt, Vector-valued Laplace transforms and Cauchy problems, Israel Journal of Mathematics 59 (1987), no. 3, 327-352.

[3] T. A. Burton and C. Kirk, A fixed point theorem of Krasnoselskii-Schaefer type, Mathematische Nachrichten 189 (1998), 23-31. 
[4] L. Byszewski and H. Akca, On a mild solution of a semilinear functional-differential evolution nonlocal problem, Journal of Applied Mathematics and Stochastic Analysis 10 (1997), no. 3, 265271.

[5] G. Da Prato and E. Sinestrari, Differential operators with nondense domain, Annali della Scuola Normale Superiore di Pisa. Classe di Scienze. Serie IV 14 (1987), no. 2, 285-344 (1988).

[6] B. C. Dhage and J. Henderson, Existence theory for nonlinear functional boundary value problems, Electronic Journal of Qualitative Theory of Differential Equations 2004 (2004), no. 1, 1-15.

[7] K. Ezzinbi and J. H. Liu, Nondensely defined evolution equations with nonlocal conditions, Mathematical and Computer Modelling 36 (2002), no. 9-10, 1027-1038.

[8] D. J. Guo and V. Lakshmikantham, Nonlinear Problems in Abstract Cones, Notes and Reports in Mathematics in Science and Engineering, vol. 5, Academic Press, Massachusetts, 1988.

[9] S. Heikkilä and V. Lakshmikantham, Monotone Iterative Techniques for Discontinuous Nonlinear Differential Equations, Monographs and Textbooks in Pure and Applied Mathematics, vol. 181, Marcel Dekker, New York, 1994.

[10] M. Kamenskii, V. Obukhovskii, and P. Zecca, Condensing Multivalued Maps and Semilinear Differential Inclusions in Banach Spaces, de Gruyter Series in Nonlinear Analysis and Applications, vol. 7, Walter de Gruyter, Berlin, 2001.

[11] H. Kellerman and M. Hieber, Integrated semigroups, Journal of Functional Analysis 84 (1989), no. $1,160-180$.

M. Belmekki: Laboratoire de Mathématiques, Université de Sidi Bel Abbès, BP 89,

Sidi Bel Abbès 22000, Algeria

E-mail address: m.belmekki@caramail.com

M. Benchohra: Laboratoire de Mathématiques, Université de Sidi Bel Abbès, BP 89,

Sidi Bel Abbès 22000, Algeria

E-mail address: benchohra@yahoo.com

S. K. Ntouyas: Department of Mathematics, University of Ioannina, 45110 Ioannina, Greece

E-mail address: sntouyas@uoi.gr 